\title{
Close encounters of the golden and silver ratios
}

\author{
Robin James Spivey \\ GeoVista, 10 Glan Y Môr, Glan Conwy, LL28 5SP, United Kingdom \\ e-mail: y.gofodegmail.com
}

Received: 31 January 2019

Revised: 12 July 2019

Accepted: 14 July 2019

\begin{abstract}
What are the nearest approaches of the natural powers of two irrational numbers, allowing for arbitrarily large exponents? In the case of the first two metallic means, a definitive answer to this challenging question lies within reach. Despite the small magnitude of the golden ratio, $\phi=(1+\sqrt{5}) / 2 \approx 1.618$, and the silver ratio, $\delta_{s}=1+\sqrt{2} \approx 2.414$, the integers approximated by their powers, namely the Lucas $\left(L_{m} \approx \phi^{m}\right)$ and Pell-Lucas $\left(U_{n} \approx \delta_{s}^{n}\right)$ numbers, never coincide except in trivial cases for which $m=0$. The equation $L_{m}=U_{n} \pm 1$ has only four solutions for $m>0, n>0$. The largest such encounter arises between $L_{11}=199$ and $U_{6}=198$ whilst the separation between larger pairings, $m>11$ and $n>6$, always exceeds 42 .
\end{abstract}

Keywords: Lucas series, Pell-Lucas series, Golden ratio, Silver ratio, Metallic means.

2010 Mathematics Subject Classification: 11B39, 14G05, 11D25.

\section{Introduction}

What is to prevent the natural powers of two irrational numbers $\alpha$ and $\beta$ from possessing very similar values? For example, if $\alpha=3 \pi / 4$ and $\beta=\exp (1)$, then although $\alpha^{7} \approx 403.16$ and $\beta^{6} \approx 403.43$ are of comparable magnitude, other exponents could potentially achieve superior agreement. However, identifying a single example might well prove difficult, even if there happens to be an infinite number of solutions.

This work will show that for a certain choice of $\alpha$ and $\beta$ possessing certain properties, a global analysis may be undertaken to determine the closest encounters between $\alpha^{m}$ and $\beta^{n}$ involving all permutations of the natural numbers $m$ and $n$. The problem has some similarity to Catalan's conjecture, proven by Preda Mihăilescu in 2002, that $3^{2}=9$ and $2^{3}=8$ differ by less than any other pairing of the powers of two natural numbers [3]. Unlike Catalan's 1844 conjecture, 
the present problem concerns the natural powers of irrational numbers. Nevertheless, works exploring the intersections of integer sequences also have some relevance here $[1,4]$.

The metallic means are important geometrically [5] and correspond to positive solutions of the equation $M_{h}-1 / M_{h}=h$ where $h \in \mathbb{N}$ and $M_{h}=\left(h+\sqrt{h^{2}+4}\right) / 2$. These irrational numbers all exceed unity and their natural powers approximate natural numbers, especially as the exponents increase in value [12]. For example, $M_{7}^{j}$ differs from an integer by less than $10^{-9}$ for $j>8$. The golden ratio, $\phi=M_{1}=1 / 2+\sqrt{5} / 2$, and the silver ratio, $\delta_{s}=M_{2}=1+\sqrt{2}$, are distinguished by elegant periodic continued fractions and infinitely nested radicals:

$$
\begin{array}{cc}
\phi=1+\frac{1}{1+\frac{1}{1+\cdots}}, & \delta_{s}=2+\frac{1}{2+\frac{1}{2+\cdots}} \\
\phi=\sqrt{1+\sqrt{1+\sqrt{1+\sqrt{\cdots}}}}=\sqrt{1+\phi}, & \delta_{s}=\sqrt{1+\delta_{s}+\sqrt{1+\delta_{s}+\sqrt{\cdots}}}=\sqrt{1+2 \delta_{s}}
\end{array}
$$

As is well-known, if $m>1$ where $m$ is a natural number, $\phi^{m}$ approximates the terms of the Lucas series, $L_{m}$, defined by $L_{0}=2, L_{1}=1$ and the recurrence relation $L_{m}=L_{m-1}+L_{m-2}$. Likewise, for $n>1$, where $n$ is a natural number, $\delta_{s}^{n}$ approximates the terms of the Pell-Lucas series, $U_{n}$, defined by $U_{0}=2, U_{1}=2$ and the recurrence relation $U_{n}=2 U_{n-1}+U_{n-2}$. As $m$ and $n$ increase, the errors in the approximations $\phi^{m} \approx L_{m}$ and $\delta_{s}^{n} \approx U_{n}$ diminish exponentially.

Lucas numbers [7] are closely related to the Fibonacci numbers, $F_{m}$, which follow the same recurrence relation but have different seed values [6]. Terms sharing the same index obey the identity $L_{m}^{2}=5 F_{m}^{2}+4 \cdot(-1)^{m}$. Similarly, the Pell-Lucas numbers [9], also known as the companion Pell numbers, are closely related to the Pell numbers, $P_{n}$, which follow the same recurrence relation with different seed values [8]. Terms sharing the same index drawn from these series obey the identity $U_{n}^{2}=8 P_{n}^{2}+4 \cdot(-1)^{n}$. Whereas the quotients $L_{m} / F_{m}$ provide optimal approximations to $\sqrt{5}$, the quotients $U_{n} / P_{n}$ provide optimal approximations to $\sqrt{8}$.

The terms of the Lucas series may be obtained directly from the golden ratio via the identity $L_{m}=\phi^{m}+(-1)^{m} \phi^{-m}$. Likewise, the terms of the Pell-Lucas series may be obtained directly from the silver ratio via the identity $U_{n}=\delta_{s}^{n}+(-1)^{n} \delta_{s}^{-n}$. To summarise,

$$
\begin{aligned}
& L_{m}=\sqrt{5 F_{m}^{2}+4 \cdot(-1)^{m}}=\phi^{m}+(-1)^{m} \phi^{-m}, \\
& U_{n}=\sqrt{8 P_{n}^{2}+4 \cdot(-1)^{n}}=\delta_{s}^{n}+(-1)^{n} \delta_{s}^{-n} .
\end{aligned}
$$

Furthermore, both $L_{m}$ and $U_{n}$ are expressible using hyperbolic functions,

$$
\begin{array}{rlrl}
L_{m} & =\phi^{m}+\phi^{-m}=2 \cosh (m \ln \phi)=2 \cosh \left(m \cdot \operatorname{arcsinh} \frac{1}{2}\right) & & m \text { even, } \\
L_{m} & =\phi^{m}-\phi^{-m}=2 \sinh (m \ln \phi)=2 \sinh \left(m \cdot \operatorname{arcsinh} \frac{1}{2}\right) & & m \text { odd } \\
U_{n} & =\delta_{s}^{n}+\delta_{s}^{-n}=2 \cosh \left(n \ln \delta_{s}\right)=2 \cosh (n \cdot \operatorname{arcsinh} 1) & & n \text { even, } \\
U_{n}=\delta_{s}^{n}-\delta_{s}^{-n}=2 \sinh \left(n \ln \delta_{s}\right)=2 \sinh (n \cdot \operatorname{arcsinh} 1) & & n \text { odd }
\end{array}
$$

The question addressed here is whether the nearest encounters between natural powers of the golden and silver ratios, $\phi^{m}$ and $\delta_{s}^{n}$, may be identified for $m \geq 1$ and $n \geq 1$. The analysis shall 
make use of the close ties between $\phi^{m}$ and the Fibonacci/Lucas series and between $\delta_{s}^{n}$ and the Pell/Pell-Lucas series. It shall be shown that the only Lucas number present in the Pell-Lucas series is $L_{0}=2$, corresponding to a trivial index/exponent of zero. The work shall then proceed to exhaustively identify solutions to the equation $L_{m}=U_{n} \pm 1$. Finally, the analysis shall be extended to encompass small offsets other than unity, $L_{m}=U_{n} \pm k$ where $k \ll L_{m}, U_{n}$.

Whilst the natural powers of the golden and silver ratios approximate integers, those integers maintain their distance from one another even when arbitrarily large exponents are considered. It shall be shown that just four solutions exist to the equation $L_{m}=U_{n} \pm 1$, the largest being $L_{11}=U_{6}+1$, which involves the numbers 198 and 199. This result is rather remarkable when one realises that all solutions to $L_{m}=U_{n} \pm k$ involve terms smaller than $L_{11}$ and $U_{6}$ if $2 \leq k \leq 42$.

\section{Assessing solutions to $\phi^{m} \approx \delta_{s}^{n}$ via continued fractions}

If $L_{m}$ and $U_{n}$ are similar in value with $m \gg 1$ and $n \gg 1$, then $\phi^{m} \approx \delta_{s}^{n}$, from which $m \ln \phi \approx n \ln \delta_{s}$. Consequently, the rational fraction $m / n$ must approximate $\ln \delta_{s} / \ln \phi$, an irrational quantity. Since $\phi$ and $\delta_{s}$ are algebraic numbers exceeding unity, both $\ln \delta_{s} \approx 0.88137$ and $\ln \phi \approx 0.48121$ are transcendental. Were it true that $m \ln \phi$ and $n \ln \delta_{s}$ were equal it would follow that $\ln \phi^{m}=\ln \delta_{s}^{n}$ and hence $(1 / 2+\sqrt{5} / 2)^{m}=(1+\sqrt{2})^{n}$. However, $\sqrt{5}$ and $\sqrt{2}$ belong to different quadratic fields. Therefore, the presumption that $m \ln \phi=n \ln \delta_{s}$ is incorrect, which implies that $\ln \delta_{s} / \ln \phi$ must be irrational. Thus, the continued fraction of $\ln \delta_{s} / \ln \phi$, which may also be expressed as $\sinh ^{-1}(1) / \sinh ^{-1}(1 / 2)$, possesses infinitely many terms, the first 67 being:

$$
\begin{array}{r}
\ln \delta_{s} / \ln \phi=[1 ; 1,4,1,14,1,12,1,6,1,7,4,4,1,2,7,11,6,5,1,21,1,4 \\
1,1,5,1,2,1,2,8,1,5,2,1,1,1,1,2,3,1,166,2,2,2,1,1 \\
8,1,8,4,1,1,1,2,2,1,1,6,4,1,3,2,1,1,1,2170, \ldots]
\end{array}
$$

The 42-nd and 67-th terms are particularly large (166 and 2170). However, the following 800 terms are all less than a thousand. Truncation of an infinitely continued fraction yields optimal rational approximations, obviating the need to consider alternatives of inferior quality. Table 1 presents the first 18 convergents (numbered $W$ ) along with those obtained by truncation at appealing locations after 41 and 66 terms.

The most accurate convergents presented in the table only achieve agreement between $L_{m}$ and $U_{n}$ in a few leading digits, a minuscule fraction of the total digits. Approximately speaking, the $W$-th convergent typically achieves agreement between $L_{m}$ and $U_{n}$ in just the first $W / 2$ decimal digits, whereas $L_{m}$ and $U_{n}$ have around $0.209 m \approx 0.383 n$ decimal digits which, since $W \sim \ln (m)$, is roughly $\exp (W) / 5$. Thus, the fraction of correct leading decimal digits, $5 W / 2 \exp (W)$, declines exponentially, tending to zero as $W \rightarrow \infty$. At the 66 -th convergent, $5 W / 2 \exp (W)$ is already smaller than $10^{-26}$.

Moreover, since the next 800 terms of the continued fraction expansion are no larger than 2170 (the 67-th term) solutions in which a substantial fraction of the digits of $L_{m}$ and $U_{n}$ agree may be confidently dismissed for $67 \leq W \leq 867$. Beyond $m \approx \exp (867)$, one finds that both $L_{m}$ and $U_{n}$ exceed $10^{\left(10^{376}\right)}$, far larger than a googolplex. There is therefore a strong suspicion that 
$L_{m}=U_{n} \pm k$ has only a finite number of solutions for $k \ll L_{m}, U_{n}$ and that the indices of any solutions will most probably be very small. The remainder of this work focuses on confirming the validity of this suspicion, and compiling an exhaustive list of solutions for $0 \leq k \leq 16$.

\begin{tabular}{|c|l|l|c|c|c|}
\hline$W$ & $m$ & $n$ & $\left|L_{m}-U_{n}\right|$ & $L_{m}, U_{n}$ & Correct digits \\
\hline \hline 1 & 1 & 1 & 1 & 1,2 & $1 / 1$ \\
2 & 2 & 1 & 1 & 3,2 & $1 / 1$ \\
3 & 9 & 5 & 6 & 76,82 & $1 / 2$ \\
4 & 11 & 6 & 1 & 199,198 & $3 / 3$ \\
5 & 163 & 89 & $5.5 \times 10^{31}$ & $1.2 \times 10^{34}$ & $2 / 34$ \\
6 & 174 & 95 & $8.5 \times 10^{32}$ & $2.3 \times 10^{36}$ & $3 / 36$ \\
7 & 2251 & 1229 & $10^{467}$ & $10^{470}$ & $3 / 470$ \\
8 & 2425 & 1324 & $10^{502}$ & $10^{507}$ & $5 / 507$ \\
9 & 16801 & 9173 & $10^{3507}$ & $10^{3511}$ & $4 / 3511$ \\
10 & 19226 & 10497 & $10^{4013}$ & $10^{4018}$ & $5 / 4018$ \\
11 & 151383 & 82652 & $10^{31631}$ & $10^{31637}$ & $6 / 31637$ \\
12 & 624758 & 341105 & $10^{130561}$ & $10^{130567}$ & $6 / 130567$ \\
13 & 2650415 & 1447072 & $10^{553897}$ & $10^{553904}$ & $7 / 553904$ \\
14 & 3275173 & 1788177 & $10^{684464}$ & $10^{684471}$ & $7 / 684471$ \\
15 & 9200761 & 5023426 & $10^{1922837}$ & $10^{1922845}$ & $8 / 1922845$ \\
16 & 67680500 & 36952159 & $10^{14144379}$ & $10^{14144388}$ & $9 / 14144388$ \\
17 & 753686261 & 411497175 & $10^{157511103}$ & $10^{157511113}$ & $10 / 157511113$ \\
18 & 4589798066 & 2505935209 & $10^{959211057}$ & $10^{959211067}$ & $10 / 959211067$ \\
\hline 41 & 4260682912080024725 & 2326245113670320106 & $10^{9 \times 10^{17}-21}$ & $10^{9 \times 10^{17}}$ & $21 / 10^{9 \times 10^{17}}$ \\
\hline 66 & $436211221992938-$ & $238162342663948-$ & $10^{9 \times 10^{28}-33}$ & $10^{9 \times 10^{28}}$ & $33 / 10^{9 \times 10^{28}}$ \\
& -874024224645141 & -622139152815172 & & & \\
\hline
\end{tabular}

Table 1 . The $W$-th convergents for $m / n \approx \ln \left(\delta_{s}\right) / \ln (\phi) \approx 1.831570923907314796$

\section{Intersections of $L_{m}$ and $U_{n}$}

The Lucas series and the Pell-Lucas series are the best known examples of Lucas V-Sequences $V_{n}(P, Q)$ which obey the recurrence relation $V_{n+2}=P \cdot V_{n+1}-Q \cdot V_{n}$. Since these sequences all commence with $V_{0}(P, Q)=2$ and $V_{1}(P, Q)=P$ the equality between $L_{0}$ and $U_{0}$ is, by definition, unavoidable. As $U_{1}=U_{0}$ there is also a semi-trivial solution, $L_{0}=U_{1}$. The remaining question addressed in this section is whether non-trivial solutions exist to the equation $L_{m}=U_{n}$ for which $m>0$ and $n>0$.

Consider the identities (1) and (2). If $m$ and $n$ share the same parity, i.e. $m \equiv n(\bmod 2)$, then $L_{m}=U_{n}$ implies $5 F_{m}^{2}=8 P_{n}^{2}$. This is satisfied for $F_{0}=P_{0}=0$, alluding to the trivial solution at $L_{0}=U_{0}=2$, but if $P_{n} \neq 0$, then $F_{m} / P_{n}$ would have to equal the irrational quantity $\sqrt{8 / 5}$. As $F_{m} / P_{n}$ is rational, $\sqrt{F_{m}^{2} / P_{n}^{2}} \neq \sqrt{8 / 5}$, precluding non-trivial solutions to $L_{m}=U_{n}$ if $m \neq 0$ and $n \neq 0$ share the same parity. 
If, on the other hand, $m$ and $n$ have opposite parity, then $5 F_{m}^{2}=8 P_{n}^{2} \pm 8$. Whereas $8\left(P_{n}^{2} \pm 1\right)$ is always a multiple of eight, $5 F_{m}^{2}$ is only a multiple of eight if $m \equiv 0(\bmod 6)$. Therefore, if $m$ is odd and $n$ is even, then $L_{m} \neq U_{n}$.

This leaves open the possibility of solutions to $\phi^{m}+\phi^{-m}=\delta_{s}^{n}-\delta_{s}^{-n}$ for even $m$ and odd $n$. The existence here of a semi-trivial solution, $\phi^{0}+\phi^{0}=\delta_{s}-1 / \delta_{s}=2$, prohibits congruences from ruling out all solutions. For example, although comparison of $5 F_{m}^{2}+4$ and $8 P_{n}^{2}-4$ modulo 6 and 9 reveals that $m \equiv 0(\bmod 12)$ and $n \equiv \pm 1(\bmod 12)$, the door remains ajar to undiscovered solutions.

If $m$ is even and $n$ is odd, then $5 F_{m}^{2}=8 P_{n}^{2}-8$. Here, the quantity $P_{n}^{2}-1$ must be a multiple of both 2 and 5 , so $P_{n}^{2}-1=10 \square$, where $\square$ denotes the square of an integer. One then arrives at the Pell equation $a^{2}-10 b^{2}=1$. With $\sqrt{10}=[3 ; \overline{6}] \approx 19 / 6$, the fundamental solution is $\left(a_{1}=19\right.$, $\left.b_{1}=6\right)$ which is unacceptable since 19 is not a Pell number and $\sqrt{8 \times(19-1)(19+1) / 5}=24$ is not a Fibonacci number. An infinite number of alternative solutions exist for $a$ and $b$ but it is the intersections between $a$ and the Pell series that are of interest. Thus, the original task of identifying solutions to $L_{m}=U_{n}$ has merely been transformed into another problem of comparable complexity. Whereas methods for solving simultaneous Pellian equations are known, they are computationally intensive [11].

If $m$ is even, then $L_{m}=\phi^{m}+\phi^{-m}$. From (2), $U_{n}^{2}=8 P_{n}^{2}-4$ for odd $n$, and thus if $L_{m}=U_{n}$ for even $m$ and odd $n$,

$$
\begin{array}{r}
\phi^{m}+\phi^{-m}=\sqrt{8 P_{n}^{2}-4} \\
\phi^{2 m}+2+\phi^{-2 m}=8 P_{n}^{2}-4 \\
\left(\phi^{2 m}\right)^{2}+\left(6-8 P_{n}^{2}\right) \phi^{2 m}+1=0 \\
\phi^{2 m}=\left(8 P_{n}^{2}-6 \pm \sqrt{\left(8 P_{n}^{2}-6\right)^{2}-4}\right) / 2 \\
\phi^{2 m}=4 P_{n}^{2}-3 \pm \sqrt{\left(4 P_{n}^{2}-3\right)^{2}-1}
\end{array}
$$

Since $\phi$ has a $\sqrt{5}$ component, if $m>0$, then $\phi^{2 m}$ also has a $\sqrt{5}$ component. Therefore, the term under the radical must be a multiple of a square,

$$
\begin{aligned}
\left(4 P_{n}^{2}-3\right)^{2}-1 & =5 \square \\
8\left(2 P_{n}^{4}-3 P_{n}^{2}+1\right) & =5 \square \\
8\left(2 P_{n}^{2}-1\right)\left(P_{n}^{2}-1\right) & =5 \square
\end{aligned}
$$

This is a quartic equation in $P_{n}$ with a finite number of integer solutions irrespective of whether those solutions correspond to Pell numbers. The $2 P_{n}^{2}-1$ term cannot be zero since that would require $P_{n}=1 / \sqrt{2}$, which is irrational. However, if $P_{n}^{2}=1$, the equation simplifies dramatically, yielding an acceptable solution alluding to the $L_{0}=U_{1}$ solution for $L_{m}=U_{n}$. Some further simplification is possible by noting that $2 \sqrt{2 / 5} \times \sqrt{\left(2 P_{n}^{2}-1\right)\left(P_{n}^{2}-1\right)} \in \mathbb{Z}$. From this, one finds that $\left(2 P_{n}^{2}-1\right)\left(P_{n}^{2}-1\right)$ must be divisible by 2 and 5 , giving $\left(2 P_{n}^{2}-1\right)\left(P_{n}^{2}-1\right)=10 \square$. If $P_{n}^{2} \neq 1$, then this remains a quartic Diophantine equation which, based on the factorisation presented, is unlikely to possess any other solutions.

For even $m$ the Lucas numbers obey the identity $L_{m}=L_{m / 2}^{2}+2$ if $m / 2$ is odd and, for even $m / 2, L_{m}=L_{m / 2}^{2}-2$. Thus, if $U_{n}$ and $L_{m}$ coincide, they must both be two distant from a square. 
Whilst this condition is invariably satisfied for $L_{m}$, it is not for $U_{n}$. Recently, near-multiples of squares have been identified for the Fibonacci, Lucas, Pell and Pell-Lucas integer sequences [2]. The findings, drawing upon results obtained via computer algebra, indicate that if $n$ is odd, then $U_{n} \pm 2$ is only square for $U_{1}=2$ or $U_{3}=14$. Whilst $U_{1}=L_{0}=2$, the term $U_{3}=14$ does not coincide with any Lucas number. Hence, $L_{m}=U_{n}$ is only trivially satisfied for $m=0$ and $n=0,1\left(L_{0}=U_{0}=U_{1}=2\right)$.

Due to the difficulty in assessing the reliability of mathematical results resting upon results obtained by computer methods, another proof that $L_{m} \neq U_{n}$ shall be provided. For the odd $m$, even $n$ branch, $L_{m}=U_{n}$ is equivalent to $\phi^{m}-\phi^{-m}=\delta_{s}^{n}+\delta_{s}^{-n}$. Since it has already been demonstrated that this has no solutions,

$$
\phi^{m}-\delta_{s}^{n} \neq \phi^{-m}+\delta_{s}^{-n}
$$

This inequality may be extended to even $m$ and odd $n$ as follows. If $m$ is odd, then $\phi^{m}-\phi^{-m} \in \mathbb{Z}$ but if $m$ is an even number larger than zero, then $\phi^{m}-\phi^{-m}$ is not an integer and, belonging to the quadratic field $\mathbb{Q}(\sqrt{5})$, it has a non-zero $\sqrt{5}$ component. Similarly, if $n$ is even, then $\delta_{s}^{n}+\delta_{s}^{-n} \in \mathbb{Z}$ but if $n$ is odd, then $\delta_{s}^{n}+\delta_{s}^{-n}$ is not an integer and, belonging to the quadratic field $\mathbb{Q}(\sqrt{2})$, it has a non-zero $\sqrt{2}$ component. Thus, if $m>0$, both $\phi^{m}-\phi^{-m}$ and $\delta_{s}^{n}+\delta_{s}^{-n}$ are irrational and belong to different quadratic fields, prohibiting equality between the two. Thus, the inequality $\phi^{m}-\delta_{s}^{n} \neq \phi^{-m}+\delta_{s}^{-n}$ holds for all $n \geq 0$ and $m>0$, not merely odd $m$ and even $n$.

If $L_{m}=U_{n}$ and $m$ is even and $n$ is odd, then $\phi^{m}+\phi^{-m}=\delta_{s}^{n}-\delta_{s}^{-n}$ and hence,

$$
\delta_{s}^{n}-\phi^{m}=\phi^{-m}+\delta_{s}^{-n}
$$

Whereas squaring the previous result gives $\left(\phi^{m}-\delta_{s}^{n}\right)^{2} \neq\left(\phi^{-m}+\delta_{s}^{-n}\right)^{2}$, squaring the present equation yields $\left(\delta_{s}^{n}-\phi^{m}\right)^{2}=\left(\phi^{-m}+\delta_{s}^{-n}\right)^{2}$. Since $\left(\delta_{s}^{n}-\phi^{m}\right)^{2}$ and $\left(\phi^{m}-\delta_{s}^{n}\right)^{2}$ are identical then, unless $m=0$, a contradiction arises. Therefore, any solution to the equation $L_{m}=U_{n}$ must involve $m=0$. Thus, when $m$ and $n$ are of opposite parity, $L_{0}=U_{1}=2$ is the only permissible solution. It follows that the equation $\cosh (m \ln \phi)=\sinh \left(n \ln \delta_{s}\right)$ can only be satisfied if $m=0$ and $n=1$. The corresponding result for odd $m$ and even $n$ is that $\sinh (m \ln \phi) \neq \cosh \left(n \ln \delta_{s}\right)$.

In conclusion, the only solutions to the equation $L_{m}=U_{n}$ are the trivial case $L_{0}=U_{0}=2$ and the semi-trivial case, $L_{0}=U_{1}=2$. The absence of non-trivial solutions is not altogether surprising given that $m / n$ must approximate $\ln \delta_{s} / \ln \phi$ to increasingly high precision as $m$ and $n$ increase.

\section{Intersections of $L_{m}$ and $U_{n} \pm 1$}

Aside from the (unavoidable) initial term, it has been shown there is no Lucas number present in the Pell-Lucas series. Hence, $\phi^{m}$ and $\delta_{s}^{n}$ never approximate the same integer for $m>1$. Nevertheless, this does not preclude encounters between $\phi^{m}$ and $\delta_{s}^{n}$ closer than unity, even for arbitrarily large exponents. 
Since $L_{m}=\phi^{m}+\phi^{-m}$ for even $m$ and $L_{m}=\phi^{m}-\phi^{-m}$ for odd $m$, solutions bifurcate according to the parity of $m$. Similarly, since $U_{n}=\delta_{s}^{n}+\delta_{s}^{-n}$ for even $n$ and $U_{n}=\delta_{s}^{n}-\delta_{s}^{-n}$ for odd $n$, solutions bifurcate according to the parity of $n$. It is therefore convenient to subdivide the question of whether solutions exist to $L_{m}=U_{n} \pm 1$ according to the parity of $m$ and $n$. This yields four distinct sub-cases which shall be analysed separately.

\subsection{Solutions to $L_{m}=U_{n} \pm 1$ for $m$ and $n$ both even}

With $m$ and $n$ even, both $m / 2$ and $n / 2$ are integers and the following identities hold:

$$
\begin{array}{r}
L_{m}=L_{m / 2}^{2} \pm 2 \\
U_{n}=U_{n / 2}^{2} \pm 2
\end{array}
$$

If $L_{m}=U_{n} \pm 1$, then $L_{m / 2}^{2}$ and $U_{n / 2}^{2}$ cannot differ by more than five and so:

$$
\left|L_{m / 2}^{2}-U_{n / 2}^{2}\right|=\left|L_{m / 2}-U_{n / 2}\right| \times\left(L_{m / 2}+U_{n / 2}\right) \leq 5
$$

Previous analysis has shown that $L_{m} \neq U_{n}$ for $m, n>0$ and thus $L_{m / 2} \neq U_{n / 2}$. It follows that $\left|L_{m / 2}-U_{n / 2}\right|$ cannot be less than one and therefore $L_{m / 2}+U_{n / 2} \leq 5$. This can only be satisfied if $m / 2 \leq 2$ and $n / 2 \leq 1$. Aside from the semi-trivial pairing ( $L_{2}=3, U_{0}=2$ ), it is easily confirmed that the only solution compatible with these constraints is ( $L_{4}=7, U_{2}=6$ ), which abides by the condition $m, n \geq 1$.

\subsection{Solutions to $L_{m}=U_{n} \pm 1$ for odd $m$ and even $n$}

As for the even $m$, even $n$ case (19) still holds true here. Therefore, if $L_{m}=U_{n} \pm 1$, there must be a perfect square at either $L_{m} \pm 1$ or $L_{m} \pm 3$. Since $U_{n / 2} \equiv 2(\bmod 4)$, that square will be congruent to $4(\bmod 8)$. Furthermore, from (2) we have $U_{n / 2}^{2}=8 P_{n / 2}^{2} \pm 4$ and $8 P_{n / 2}^{2}$ is twice the square of an even number. Invariably, $U_{n}$ falls between $U_{n / 2}^{2}$ and $8 P_{n / 2}^{2}$ here. If $L_{m}=U_{n} \pm 1$, then $L_{m}$ must also lie between $U_{n / 2}^{2}$ and $8 P_{n / 2}^{2}$. Hence, either $L_{m} \pm 1$ or $L_{m} \pm 3$ must be twice the square of an even number.

It is known [2] that the only Lucas numbers 1 or 3 distant from twice a square are $L_{0}=2$, $L_{1}=1, L_{2}=3, L_{3}=4, L_{4}=7$ and $L_{11}=199$. The same work also showed that, of these, the only Lucas numbers 1 or 3 distant from twice a square are $L_{1}=1, L_{2}=3, L_{4}=7$ and $L_{11}=199$. Since $m$ must be odd, the only relevant solutions are the semi-trivial solution $\left(L_{1}=1, U_{0}=2\right)$ which is of no interest here as the index of the Pell-Lucas term is zero, along with a remarkably large non-trivial solution at $\left(L_{11}=199, U_{6}=198\right)$. This result was only to be anticipated based on the fourth convergent of $\ln \delta_{s} / \ln \phi$ presented in Table 1.

\subsection{Solutions to $L_{m}=U_{n} \pm 1$ for even $m$ and odd $n$}

As for the even $m$, even $n$ case, (18) holds true again here. Therefore, if $L_{m}=U_{n} \pm 1$, a perfect square must be present either at $U_{n} \pm 1$ or at $U_{n} \pm 3$. Furthermore, the identity $L_{m / 2}^{2}=5 F_{m / 2}^{2} \pm 4$ 
applies so that $L_{m}$ invariably falls between $L_{m / 2}^{2}$ and $5 F_{m / 2}^{2}$. If $L_{m}=U_{n} \pm 1$, then $U_{n}$ must also lie between $L_{m / 2}^{2}$ and $5 F_{m / 2}^{2}$. Hence, either $U_{n} \pm 1$ or $U_{n} \pm 3$ must be a perfect square.

It is known [2] that the only Pell-Lucas numbers 1 or 3 distant from a square are $U_{0}=2$, $U_{1}=2, U_{2}=6$ and $U_{5}=82$. It is, then, a trivial task to investigate $U_{1}$ and $U_{5}$, the only terms with an odd index. Whereas there is no Lucas number adjacent to $U_{5}, L_{2}=3$ is adjacent to $U_{1}=2$. Therefore, only one acceptable solution exists for even $m$ and odd $n,\left(L_{2}=3, U_{1}=2\right)$, corresponding to the second convergent of Table 1.

\subsection{Solutions to $L_{m}=U_{n} \pm 1$ for $m$ and $n$ both odd}

With both $m$ and $n$ odd, neither of the identities $L_{m}=L_{m / 2}^{2} \pm 2$ and $U_{n}=U_{n / 2}^{2} \pm 2$ apply here so there can be no expectation that either $L_{m}$ or $U_{n}$ is adjacent to a square or the multiple of a square. Despite the seeming freedom this affords, there is only one known solution for this branch, $L_{1}=U_{1}-1$. Its simplicity may be hinting that any further solutions must comply with some stringent but hitherto unknown constraint.

Since the existence of at least one solution is not in doubt, arguments based on congruences cannot completely rule out other solutions. Whilst it is straightforward to show that $m$ and $n$ must be congruent to \pm 1 modulo 12,24 , or even 7920 , it is uncertain whether a path could be forged allowing such results to be extended to arbitrarily large moduli. Moreover, resistance to congruence analysis extends to many equivalent formulations of the case in hand, obtained by exploiting the panoply of identities the Lucas sequences give rise to, such as $U_{n}=L_{v} L_{v+1}$ where $v=(m-1) / 2$.

Nevertheless, congruences are of some avail in simplifying the problem. If $m$ is a multiple of three then $L_{m}$ is even and so $L_{m} \pm 1$ is odd, prohibiting solutions. If $m$ is not a multiple of 3 then $\left(\left(L_{m}-1\right)^{2}+4\right) / 2$ is always congruent to $2(\bmod 4)$, a quadratic non-residue. Therefore, $L_{m}-1$ cannot equal $U_{n}$ since, via (2), that would require $\left(\left(L_{m}-1\right)^{2}+4\right) / 2=\left(U_{n}^{2}+4\right) / 2=\left(2 P_{n}\right)^{2}=\square$. Accordingly, $L_{m} \neq U_{n}+1$ if $m$ and $n$ are odd, leaving $L_{m}=U_{n}-1$ as the only remaining possibility.

Although one cannot appeal to the presence of squares here, the identities $L_{m}=\sqrt{5 F_{m}^{2}-4}$ and $U_{n}=\sqrt{8 P_{n}^{2}-4}$ still apply to this branch. Then, using $a$ to denote $F_{m}$ and $b$ to denote $P_{n}$, the equation $L_{m}=U_{n}-1$ becomes, using (1) and (2),

$$
\begin{array}{r}
\sqrt{5 a^{2}-4}=\sqrt{8 b^{2}-4}-1, \\
5 a^{2}-4=8 b^{2}-4-2 \sqrt{8 b^{2}-4}+1, \\
25 a^{4}+64 b^{4}-80 a^{2} b^{2}-10 a^{2}+16 b^{2}+1=32 b^{2}-16, \\
25 a^{4}+64 b^{4}-80 a^{2} b^{2}-10 a^{2}-16 b^{2}+17=0 .
\end{array}
$$

It would be advantageous to simplify this quartic Diophantine equation, if at all possible. Continued fraction analysis indicates that any undiscovered solutions must correspond to large values of $L_{m}$ and $U_{n}$. Clearly, the fourth order terms, $64 b^{4}-80 a^{2} b^{2}+25 a^{4}$, will be dominant if $L_{m}$ and $U_{n}$ are very large. Conveniently, these terms are encapsulated by the square, $\left(8 b^{2}-5 a^{2}\right)^{2}$. Since $U_{n}^{2}-L_{m}^{2}=\left(U_{n}-L_{m}\right)\left(U_{n}+L_{m}\right)$ and $U_{n}-L_{m}=1$ for the solutions sought, 
$U_{n}^{2}-L_{m}^{2}=L_{m}+U_{n}$. From (2), $U_{n}^{2}=8 b^{2}-4$, and from (1), $L_{m}^{2}=5 a^{2}-4$. Thus, $U_{n}^{2}-L_{m}^{2}=8 b^{2}-5 a^{2}$, from which one obtains $\left(8 b^{2}-5 a^{2}\right)^{2}=\left(L_{m}+U_{n}\right)^{2}$.

If $c$ is defined as $8 b^{2}-5 a^{2}$ then, in the presence of a solution, $c=L_{m}+U_{n}$. The quartic expression (24) is then the sum of $c^{2}$ and lower order terms $\left(a^{2}, b^{2}\right.$ and a numerical constant). The same is true if various integer offsets are applied to $c$ prior to taking the square of the result. Therefore, the quartic may be rephrased in terms of $(c+N)^{2}$ where $N \in \mathbb{Z}$. The examples provided below correspond to $-4 \leq N \leq 4$.

$$
\begin{aligned}
& (c+4)^{2}=-30 a^{2}+80 b^{2}-1 \\
& (c+3)^{2}=-20 a^{2}+64 b^{2}-8 \\
& (c+2)^{2}=-10 a^{2}+48 b^{2}-13 \\
& (c+1)^{2}=0 a^{2}+32 b^{2}-16 \\
& (c+0)^{2}=10 a^{2}+16 b^{2}-17 \\
& (c-1)^{2}=20 a^{2}+0 b^{2}-16 \\
& (c-2)^{2}=30 a^{2}-16 b^{2}-13 \\
& (c-3)^{2}=40 a^{2}-32 b^{2}-8 \\
& (c-4)^{2}=50 a^{2}-48 b^{2}-1
\end{aligned}
$$

It is apparent that the original quartic reduces to the quadratic $(c+N)^{2}=p a^{2}+q b^{2}+r$ where, by virtue of the fact that $(c+N)^{2}-c^{2}=2 c N+N^{2}=N\left(N-10 a^{2}+16 b^{2}\right)$ and $c^{2}=10 a^{2}+16 b^{2}-17$, the integer coefficients $p, q$ and $r$ are simple functions of $N$ :

$$
p=10(1-N), \quad q=16(N+1), \quad r=N^{2}-17 .
$$

The resulting quadratic, $p a^{2}+q b^{2}+r=(c+N)^{2}$, now reads

$$
10(1-N) a^{2}+16(N+1) b^{2}+N^{2}-17=(c+N)^{2} .
$$

If $c+N=0$, then this quadratic in $N$ has the solution

$$
\begin{array}{r}
N=5 a^{2}-8 b^{2} \pm \sqrt{25 a^{2}+64 b^{4}-80 a^{2} b^{2}-10 a^{2}-16 b^{2}+17} \\
N=5 a^{2}-8 b^{2}=-c .
\end{array}
$$

The simplification to $N=-c$ occurs because the term under the radical is the original quartic expression which, providing $L_{m}=U_{n}-1$, is equal to zero.

For a valid solution, $c+N=0$ leaving a simplified equation of the form $p a^{2}+q b^{2}+r=0$ in the variables $a$ and $b$. With just three exceptions, these equations are hyperbolic. A small fraction of the equations $p a^{2}+q b^{2}+r=0$ possess integral solutions (e.g. $N=-3, N=-33$ and $N=-903)$. It is well-known that if a solution is present in equations of this form, then an infinite number of other solutions may be obtained via composition. For those equations with solutions, there may be more than one solution class present.

The only known solution for this branch, $L_{1}=U_{1}-1$, corresponds to $a^{2}=b^{2}=1$. The expression $p a^{2}+q b^{2}+r=40 a^{2}-32 b^{2}-8$ is, then, zero only if $N=-3$. This requires 
$L_{m}+U_{n}=3$ and, since $L_{m}=U_{n}-1$, one may obtain $L_{m}=1$ and $U_{n}=2$ without recourse to $L_{m}=\sqrt{5 a^{2}-4}$ and $U_{n}=\sqrt{8 b^{2}-4}$. The simplicity of the known solution is elucidated by rewriting (32) as follows:

$$
\begin{aligned}
(c-3)^{2} & =36 a^{2}+4 a^{2}-36 b^{2}+4 b^{2}-8 \\
& =(6 a-6 b)(6 a+6 b)+4\left(a^{2}+b^{2}\right)-8 .
\end{aligned}
$$

Thus, if $a^{2}=b^{2}$, this equation simplifies to $(c-3)^{2}=8\left(a^{2}-1\right)$. Of the four intersections $\{0,1,2,5\}$ between the Fibonacci and Pell series [1], $c$ is an integer only if $a^{2}=b^{2}=1$. Nevertheless, if the requirement $a^{2}=b^{2}$ is abandoned, this equation has an infinite number of solutions for some fixed value of $c$.

If $c+N \neq 0$, then the $N^{2}$ terms cancel and the equation $p a^{2}+q b^{2}+r=(c+N)^{2}$ simplifies to a linear equation, whose solution is

$$
N=\frac{10 a^{2}+16 b^{2}-c^{2}-17}{10 a^{2}-16 b^{2}+2 c} .
$$

Should $a, b$ and $c$ combine to form a solution to $L_{m}=U_{n}-1$ it is easily shown using $c=8 b^{2}-5 a^{2}$ that both the numerator and the denominator here will be zero, leaving $N$ undefined. This reflects the fact that the entire family of equations $p a^{2}+q b^{2}+r=(c+N)^{2}$ are, then, simultaneously satisfied. Of course, $c+N$ will only be zero in one instance.

Notice that $p, q$ and $r$ can alternatively be expressed in terms of $L_{m}$ and $U_{n}$ :

$$
\begin{array}{r}
p=10\left(1+L_{m}+U_{n}\right)=20\left(L_{m}+1\right)=20 U_{n}, \\
q=16\left(1-L_{m}-U_{n}\right)=-32 L_{m}=32\left(1-U_{n}\right), \\
r=\left(L_{m}+U_{n}\right)^{2}-17=\left(2 L_{m}+1\right)^{2}-17=\left(2 U_{n}-1\right)^{2}-17 .
\end{array}
$$

Accordingly, any solution to $L_{m}=U_{n}-1$, whether large or small, must satisfy

$$
\begin{array}{r}
20 U_{n} F_{m}^{2}+\left(L_{m}+U_{n}\right)^{2}=32 L_{m} P_{n}^{2}+17 \\
4 U_{n}\left(L_{m}^{2}+4\right)+\left(L_{m}+U_{n}\right)^{2}=4 L_{m}\left(U_{n}^{2}+4\right)+17 .
\end{array}
$$

The primary interest here is to uncover any large solutions to $L_{m}=U_{n}-1$, for which $N=-c=-\left(L_{m}+U_{n}\right)$. Therefore, consider (35) in the limit $N \rightarrow-\infty$. Firstly, notice that if $N^{2} \gg 17$, then $p, q$ and $r$ tend to the following values:

$$
p \rightarrow-10 N, \quad q \rightarrow 16 N, \quad r \rightarrow N^{2} .
$$

Adopting these limits as coefficients in (35) and assuming that a solution is present, which imposes the constraint $c+N=0$, yields the approximation $-10 N a^{2}+16 N b^{2}+N^{2} \approx 0$. Since $N \neq 0$, division by $N$ is permissible, yielding

$$
N \approx 10 a^{2}-16 b^{2} \approx-2\left(8 b^{2}-5 a^{2}\right) \approx-2 c .
$$

This indicates that $c+N \approx-c$, in contradiction to the condition $c+N=0$ that must be satisfied if a solution is present and $N^{2} \gg 17$. Therefore, the earlier presumption that a solution 
to the equation $L_{m}=U_{n}-1$ exists for large $L_{m}$ and $U_{n}$, or more precisely $\left(L_{m}+U_{n}\right)^{2} \gg 17$, must be incorrect. Since it is clear from the convergents of $\ln \delta_{s} / \ln \phi$ that any undiscovered solution requires $L_{m}$ and $U_{n}$ to exceed $10^{\left(9 \times 10^{28}\right)}$, if not $10^{\left(10^{376}\right)}$, numbers whose squares are enormously larger than 17, one may confidently infer the non-existence of undiscovered solutions to $L_{m}=U_{n}-1$ along this branch.

\subsection{Revisiting large solutions for all branches}

A cynic might object that results obtained via computer algebra are vulnerable to a variety of drawbacks such as undetected silicon defects, memory failures, algorithmic oversights and transcription errors. Such problems may be completely mitigated by deriving alternative proofs that, in turn, may bolster confidence in the reliability of computational approaches. Here, searching for small solutions is straightforward and the convergents of $\ln \delta_{s} / \ln \phi$ inform us that any undiscovered solutions must be extremely large. The analysis of the previous section shall now be applied to other branches to confirm the absence of any very large, hitherto undiscovered solutions to the equation $L_{m}=U_{n} \pm 1$. This is a useful first step before attempting to generalise the existing results. Consider first the case $L_{m}=U_{n} \pm 1$ where the corresponding quartics for each branch are obtained via the identities $L_{m}^{2}=5 F_{m}^{2} \pm 4$ and $U_{n}^{2}=8 P_{n}^{2} \pm 4$ so that $\sqrt{5 a^{2} \pm 4}=\sqrt{8 b^{2} \pm 4} \pm 1$ :

$$
\begin{array}{ll}
\left(8 b^{2}-5 a^{2}\right)^{2}=10 a^{2}+16 b^{2}+15 & \text { even } m, \text { even } n, \\
\left(8 b^{2}-5 a^{2}\right)^{2}=-70 a^{2}+144 b^{2}-65 & \text { even } m, \text { odd } n, \\
\left(8 b^{2}-5 a^{2}\right)^{2}=90 a^{2}-112 b^{2}-65 & \text { odd } m, \text { even } n, \\
\left(8 b^{2}-5 a^{2}\right)^{2}=10 a^{2}+16 b^{2}-17 & \text { odd } m, \text { odd } n .
\end{array}
$$

Letting $c=8 b^{2}-5 a^{2}$, the factorisation of these quartics can again be parametrised via the equation $(c+N)^{2}=p a^{2}+q b^{2}+r$ where $p, q$ and $r$ are given by

$$
\begin{array}{llll}
p=-10(N-1), & q=16(N+1), & r=N^{2}+15 & \text { even } m, \text { even } n, \\
p=-10(N+7), & q=16(N+9), & r=N^{2}-65 & \text { even } m, \text { odd } n, \\
p=-10(N-9), & q=16(N-7), & r=N^{2}-65 & \text { odd } m, \text { even } n, \\
p=-10(N-1), & q=16(N+1), & r=N^{2}-17 & \text { odd } m, \text { odd } n .
\end{array}
$$

As before, solutions for each branch correspond to $c+N=0$. Whilst $c=8 b^{2}-5 a^{2}$ once more, note that $c$ is not necessarily equal to $L_{m}+U_{n}$ here, although the two are still closely linked. For the odd $m, n$ and even $m, n$ branches, $c=\left(U_{n}-L_{m}\right)\left(L_{m}+U_{n}\right)$ which is either $L_{m}+U_{n}$ if $L_{m}+1=U_{n}$ or $-\left(L_{m}+U_{n}\right)$ if $L_{m}-1=U_{n}$. For the even $m$, odd $n$ branch, $c=\left(U_{n}-L_{m}\right)\left(L_{m}+U_{n}\right)+8$ which is either $L_{m}+U_{n}+8$ if $L_{m}+1=U_{n}$ or $8-L_{m}-U_{n}$ if $L_{m}-1=U_{n}$. For the odd $m$, even $n$ branch, $c=\left(U_{n}-L_{m}\right)\left(L_{m}+U_{n}\right)-8$ which is either $L_{m}+U_{n}-8$ if $L_{m}+1=U_{n}$ or $-\left(L_{m}+U_{n}+8\right)$ if $L_{m}-1=U_{n}$.

There is one solution for each branch. For the even $m, n$ branch, $N=L_{4}+U_{2}=13$. For the even $m$, odd $n$ branch, $N=L_{2}+U_{1}-8=-3$. For the odd $m$, even $n$ branch, $N=L_{11}+U_{6}+8=405$. Finally, for the odd $m, n$ branch, $N=-\left(L_{1}+U_{1}\right)=-3$. 
In each case, the limiting values of $p, q$ and $r$ as $N \rightarrow \pm \infty$ are unchanged and so the approximation $-10 N \mathrm{a}^{2}+16 N b^{2}+N^{2} \approx 0$ still holds and so the same contradiction arises in that $c+N \approx-c$ when in fact $c+N=0$. Again, this is unacceptable since, for all these cases, $c$ is of comparable magnitude to $L_{m}+U_{n} \gg 0$. Therefore, the presumption that a solution to $L_{m}=U_{n} \pm 1$ exists for large $L_{m}$ and $U_{n}$ must be incorrect.

\section{Results: close encounters of the first two metallic means}

The analysis technique of the previous section readily lends itself to the investigation of solutions to $L_{m} \neq U_{n} \pm k$ for relatively small integers $k$ under the assumption that $k \ll L_{m}, U_{n}$. Unsurprisingly, the same asymptotic inconsistency arises for very large terms of the Lucas and Pell-Lucas series, the inference $c+N \approx-c$ when in fact $c+N=0$. Thus, with the scaling of $k$ being a linear function of $L_{m}$ and $U_{n}$, arbitrarily large powers of the golden and silver ratio not only maintain their distance from each other, but their minimum separation must increase in tandem with $L_{m}$ and $U_{n}$. Consequently, the investigation of solutions to $L_{m}=U_{n} \pm 2, L_{m}=U_{n} \pm 3$ and so forth requires nothing more than the scrutiny of the smaller terms in the Lucas and PellLucas series and the first few convergents of the continued fraction of $\ln \delta_{s} / \ln \phi$, from which it is patently clear that even optimal approximations to $\ln \delta_{s} / \ln \phi$ soon fall hopelessly short of achieving equality between $L_{m}$ and $U_{n}$ after only the first six convergents. Thus, even a very conservative search need not consider more than the first 174 terms of the Lucas series and the first 95 terms of the Pell-Lucas series.

Due to the parallels between the natural powers of the golden and silver ratio and the terms of the Lucas $V$-sequences they approximate, it therefore becomes a simple matter to compile a brief and yet comprehensive list of the closest encounters between $L_{m} \approx \phi^{m}$ and $U_{n} \approx \delta_{s}^{n}$. An ordered list of the 24 closest non-trivial approaches of terms belonging to the Lucas and PellLucas series are presented in Table 2 encompassing all differences of sixteen or less, $0 \leq k \leq 16$. The first offset with no solutions is $L_{m}=U_{n} \pm 8$. Terms beyond $L_{11}$ or $U_{6}$ do not participate in solutions unless differences exceeding 42 are considered, highlighting the improbability of a solution as large as $L_{11}=199, U_{6}=198$ existing for the simplest equation with non-trivial solution, $L_{m}=U_{n} \pm 1$.

\section{Conclusion}

The natural powers of the golden and silver ratios shadow the terms of the Lucas and Pell-Lucas series. The terms of the two series only coincide in trivial cases, both involving the Lucas term, $L_{0}=2$. This is inevitable as all Lucas $V$-sequences commence with the number two. It has been shown here that the equation $L_{m}=U_{n} \pm 1$ has four non-trivial solutions, one for each parity permutation of $m$ and $n$. The largest such solution, $\left(L_{11}=199, U_{6}=198\right)$, is considerably larger than any other.

With the deviation between larger powers of the golden and silver ratios and the integers decaying exponentially as $m$ and $n$ increase, the four solutions to $L_{m}=U_{n} \pm 1$ draw attention 


\begin{tabular}{|c|c|}
\hline Equation & Solution(s) \\
\hline \hline$L_{m}=U_{n}$ & Trivial solutions only, $L_{0}=U_{0}=U_{1}$ \\
$L_{m}=U_{n} \pm 1$ & $\left(L_{1}=U_{1}-1\right),\left(L_{2}=U_{1}+1\right),\left(L_{4}=U_{2}+1\right),\left(L_{11}=U_{6}+1\right)$ \\
$L_{m}=U_{n} \pm 2$ & $\left(L_{3}=U_{1}+2\right),\left(L_{3}=U_{2}-2\right)$ \\
$L_{m}=U_{n} \pm 3$ & $\left(L_{2}=U_{2}-3\right),\left(L_{5}=U_{3}+3\right)$ \\
$L_{m}=U_{n} \pm 4$ & $\left(L_{6}=U_{3}+4\right)$ \\
$L_{m}=U_{n} \pm 5$ & $\left(L_{1}=U_{2}-5\right),\left(L_{4}=U_{1}+5\right),\left(L_{5}=U_{2}+5\right),\left(L_{7}=U_{4}-5\right)$ \\
$L_{m}=U_{n} \pm 6$ & $\left(L_{9}=U_{5}-6\right)$ \\
$L_{m}=U_{n} \pm 7$ & $\left(L_{4}=U_{3}-7\right)$ \\
$L_{m}=U_{n} \pm 9$ & $\left(L_{5}=U_{1}+9\right)$ \\
$L_{m}=U_{n} \pm 10$ & $\left(L_{3}=U_{3}-10\right)$ \\
$L_{m}=U_{n} \pm 11$ & $\left(L_{2}=U_{3}-11\right)$ \\
$L_{m}=U_{n} \pm 12$ & $\left(L_{6}=U_{2}+12\right)$ \\
$L_{m}=U_{n} \pm 13$ & $\left(L_{1}=U_{3}-13\right),\left(L_{8}=U_{4}+13\right)$ \\
$L_{m}=U_{n} \pm 15$ & $\left(L_{7}=U_{3}+15\right)$ \\
$L_{m}=U_{n} \pm 16$ & $\left(L_{6}=U_{1}+16\right),\left(L_{6}=U_{4}-16\right)$ \\
\hline
\end{tabular}

Table 2. The closest encounters for $L_{m}=U_{n} \pm k$ with $0 \leq k \leq 16$.

\begin{tabular}{|c|c|c|c|c|c|c|c|c|c|c|c|c|c|}
\hline Series & Term & 0 & 1 & 2 & 3 & 4 & 5 & 6 & 7 & 8 & 9 & 10 & 11 \\
\hline \hline Lucas & $L_{m}$ & 2 & 1 & 3 & 4 & 7 & 11 & 18 & 29 & 47 & 76 & 123 & 199 \\
Fibonacci & $F_{m}$ & 0 & 1 & 1 & 2 & 3 & 5 & 8 & 13 & 21 & 34 & 55 & 89 \\
\hline Pell-Lucas & $U_{n}$ & 2 & 2 & 6 & 14 & 34 & 82 & 198 & 478 & 1154 & 2786 & 6726 & 16238 \\
Pell & $P_{n}$ & 0 & 1 & 2 & 5 & 12 & 29 & 70 & 169 & 408 & 985 & 2378 & 5741 \\
\hline
\end{tabular}

Table 3. The first 12 terms of the Lucas, Pell-Lucas, Fibonacci and Pell series.

to the closest non-trivial encounters between the natural powers of the golden and silver ratios, $\delta_{s}-\phi \approx 0.796, \phi^{2}-\delta_{s} \approx 0.204, \phi^{4}-\delta_{s}^{2} \approx 1.026$ and $\phi^{11}-\delta_{s}^{6} \approx 1.01$. The adjacency of $L_{11}=199$ and $U_{6}=198$ is surprising given the absence of solutions to the equation $L_{m}=U_{n} \pm k$ for terms beyond $L_{11}$ and $U_{6}$ unless $k>42$ (in particular, $\phi^{13} \approx \delta_{s}^{7}+43$ ). Consequently, all solutions to the equation $L_{m}=U_{n} \pm k$ for $0 \leq k \leq 42$ are present in Table 3 . The analysis has also shown that the natural powers of the golden and silver ratios maintain an ever-increasing degree of separation at large $m$ and $n$. It is anticipated that the same applies to any pairing of the metallic means belonging to different quadratic fields. This excludes pairings such as $\left(M_{1}, M_{4}\right)$ or $\left(M_{3}\right.$, $M_{36}$ ), for which a list is available [10].

It is often claimed that the golden ratio is the most irrational of numbers owing to the difficulty of its accurate approximation by the ratio of two integers. According to that reasoning the silver ratio would be the second most irrational number, its continued fraction containing no terms exceeding two. Nevertheless, optimal approximations for each are readily obtained via the ratios of consecutive terms in the Fibonacci and Pell series and the metallic means are not as irrational as many imagine. Amongst the Pisot numbers, the golden ratio and the silver ratio are the smallest 
quadratic irrationals.

Unlike most irrational numbers, the propensity of the natural powers of the golden and silver ratios to cluster around the integers endows them with characteristics facilitating global analysis. Whilst these powers are attracted to certain integers, they remain irrational and, in a sense, they repel one another. The increasing separation that exists between $L_{m}$ and $U_{n}$ as $m$ and $n$ increase indicates that the terms of the continued fraction for $\ln \delta_{s} / \ln \phi$ cannot exceed certain bounds, and hence cannot be truly random.

In closing, the present analysis establishes that the largest of the non-trivial encounters between $L_{m}$ and $U_{n}$ occurs at $L_{11}=199$ and $U_{6}=198$. Since 198 and 199 correspond to the atomic mass numbers of two stable isotopes of mercury, the only metallic element which is liquid at ordinary temperatures and pressures (both STP and NTP), and ${ }^{199} \mathrm{Hg}$ is the only stable nuclide with 199 nucleons, it seems fitting to name the following theorem after the element mercury:

Mercury Theorem. For $m>0$ and $n>0$, the closest encounters between the Lucas terms $L_{m}$ and the Pell-Lucas terms $U_{n}$ correspond to four solutions of the equation $L_{m}=U_{n} \pm 1$, the largest of which occurs at $m=11, n=6$ corresponding to $L_{11}=199$ and $U_{6}=198$, a pairing exceeding any other close encounter between $L_{m}$ and $U_{n}$ for which the difference is 42 or less.

\section{Acknowledgements}

Thanks to Andrew Spivey for constructive discussions relating to the topics addressed here.

\section{References}

[1] Alekseyev, M. A. (2011). On the intersections of Fibonacci, Pell and Lucas numbers, Integers, 11(3), 239-259.

[2] Alekseyev, M. A. \& Tengely, S. (2014). On integral points on biquadratic curves and nearmultiples of squares in Lucas sequences, J. Integer Sequences, 17(2), Article 14.6.6.

[3] Catalan, E. (1844). Journal für die reine und angewandte Mathematik, 27, 192-192.

[4] Horadam, A. F. (1966). Generalizations of two theorems of K. Subba Rao, Bulletin of the Calcutta Mathematical Society, 58 (1), 23-29.

[5] Leyendekkers, J. V. \& Shannon, A. G. (2016). Some characteristics of the Golden Ratio family, Notes on Number Theory and Discrete Mathematics, 22 (3), 84-89.

[6] OEIS Foundation (2019). The Online Encyclopedia of Integer Sequences, A000045. Available online at: https://oeis.org/A000045.

[7] OEIS Foundation (2019). The Online Encyclopedia of Integer Sequences, A000032. Available online at: https: / / oeis.org/A000032. 
[8] OEIS Foundation (2019). The Online Encyclopedia of Integer Sequences, A000129. Available online at: https: / / oeis.org/A000129.

[9] OEIS Foundation (2019). The Online Encyclopedia of Integer Sequences, A002203. Available online at: https: / / oeis.org/A002203.

[10] OEIS Foundation (2019). The Online Encyclopedia of Integer Sequences, A013946. Available online at: https: / / oeis.org/A013946.

[11] Pinch, R. G. E. (1988). Simultaneous Pellian equations. Mathematical Proceedings of the Cambridge Philosophical Society, 103 (1), 35-46.

[12] Shannon, A. G. \& Leyendekkers, J. V. (2015). The Golden Ratio family and the Binet equation, Notes on Number Theory and Discrete Mathematics, 21 (2), 35-42. 